\title{
DESEMPEÑO DE NIÑOS TÍPICOS ENTRE 5 Y 7.11 AÑOS DE EDAD EN UNA SELECCIÓN DE OBSERVACIONES CLIINICAS: DATOS PRELIMINARES Y PROPIEDADES PSICOMÉTRICAS EN UNA MUESTRA CHILENA
}

\author{
THE PERFORMANCE OF TYPICAL CHILDREN BETWEEN 5 AND 7.11 YEARS IN A \\ SELECTION OF CLINICAL OBSERVATIONS: PRELIMINARY DATA AND PSYCHOMETRIC \\ PROPERTIES IN A CHILEAN SAMPLE.
}

\section{Erna Imperatore Blanche, ${ }^{1}$ Gustavo Reinoso, ${ }^{2}$ Dominique Blanche Kiefer, ${ }^{3}$ Angeles Barros ${ }^{4}$}

\begin{abstract}
Resumen
La utilización de observaciones que proporcionan información acerca del funcionamiento sensorio-motor relacionado a procesamiento sensorial es común en la práctica clínica. Un grupo de observaciones, Ilamadas observaciones clínicas por A.J. Ayres ayuda a diagnosticar disfunciones sensoriales aunque no existen normas desarrolladas en Chile, ni su validez o confiabilidad establecida estadísticamente para su utilización. Para normar estas pruebas en Chile se entrenaron 4 evaluadores quienes recolectaron datos de niños entre 5 y 7.11 años de edad: 90 típicos y 26 identificados con problemas de procesamiento sensorial. Este estudio proporciona datos preliminares sobre 5 pruebas relacionadas a control postural y planeamiento motor: equilibrio en un pie (ojos cerrados y ojos abiertos), extensión antigravitatoria, flexion antigravitatoria, secuenciación de dígitos, y diadochokinesis. Los resultados indican que estas pruebas se pueden utilizar para diagnosticar niños con disfunciones sensorio-motoras en la práctica clínica e investigación.
\end{abstract}

\section{Palabras clave:}

Evaluación diagnóstica, integración sensorial, control postural, planeamiento motor

\begin{abstract}
Clinical observations that provide information about sensory-motor performance related to sensory processing, are commonly used in clinical practice. A group of these observations, known as clinical observations by A. J. Ayres, help diagnose sensory related dysfunctions even when normative data and statistically established validity and reliability are not available for the Chilean children. In order to develop normative data, we trained 4 independent assessors who collected data on 90 typically developing children 5 to 7.11 years of age and 26 children identified as presenting with sensory processing problems. The present study provides preliminary data about 5 observations that relate to postural control and motor planning: Standing on one foot (eyes open and closed), extensión against gravity, flexion against gravity, sequential finger touching and diadochokinesis. The results of this study indicate that these observations can help diagnose children with sensory-motor dysfunctions and be utilized for clinical applications and research.
\end{abstract}

\section{Keywords:}

Diagnostic evaluation, sensory integration, postural control, motor planning

1 Associate Professor, Director Post Professional Masters. T.H Chan Division of Occupational Science and Occupational Therapy. University of Southern California, Los Angeles, USA. Email: blanche@chan.usc.edu

2 Assistant Professor and Director of Technology. Nova Southeastern University (NSU). Health Professions Division. College of Health Care Sciences. Department of Occupational Therapy-Tampa.

3232 Queen Palm Drive. Tampa, Florida 33619-1311 * Tel: (813) 574-5344 * Fax: (813) 574-5330. Email: greinoso@nova.edu

3 Therapy West, Inc. Los Angeles, USA.

$4 \quad$ Terapeuta Ocupacional Comite Academico ISChile, Practica privada. Telefono 95395239 Correo mangelesbarros@gmail.com 


\section{INTRODUCCIÓN}

Ayres originalmente describió las "observaciones clínicas" como un grupo de observaciones de habilidades motoras y comportamientos relacionados al procesamiento sensorial que ayudan a diagnosticar las dificultades de integración sensorial (Ayres, 1984). Las observaciones clínicas están estrechamente relacionados con la teoría de integración sensorial, así como con los exámenes de signos neurológicos blandos, que actualmente utilizan neurólogos y otros profesionales durante el diagnóstico de trastornos del cerebelo e incoordinación motora (Adams, et al. 1974; Fellick et al., 2001; Gustafsson, Sveding, Ericsson, Karlsson, \& Thernlund, 2010; Guftafsson, Thernlund, Ryding, Rosen \& Cerblad, 2000; Holden, Tarnowski \& Prinz, 1982; Jones \& Prior, 1985). Para los terapeutas estas observaciones, cuando son acompañadas de otros signos de procesamiento ineficiente de información sensorial, son interpretadas como una disfunción en integración sensorial.

Las observaciones estructuradas son tareas específicas que se realizan con el niño y que se utilizan para diagnosticar problemas motores (Blanche, 2002, 2010). Por ejemplo, la tarea de alternancia de movimientos de antebrazo (también conocida como diadochokinesis) y la prueba de extensión de brazos de Schilder son exámenes utilizados por neurólogos; su presencia en niños indica disfunción en el sistema nervioso central (Gillberg y Kadesjo, 2003; Schaffer, O'Connor, Schafer \& Prupil, 1984). Algunas de estas tareas han sido estudiadas por terapeutas ocupacionales en niños típicos con el propósito de proporcionar tendencias en el desarrollo que ayudan al examinador a interpretar sus resultados. Por ejemplo Frazer (1986) estudió el desempeño de niños típicos durante la flexión en supino y estableció parámetros para su interpretación en niños de 4 a 8 años de edad.

En general, la finalidad de utilizar observaciones estructuradas y no estructuradas durante el proceso de evaluación es asistir al terapeuta en la creación de una visión global de la capacidad del niño para realizar las tareas diarias y considerar los factores que pueden estar obstaculizando su rendimiento. Las observaciones tienen varias ventajas, incluyendo su flexibilidad y su propósito. La flexibilidad de las observaciones clínicas permite al terapeuta recopilar información en diferentes ámbitos y la facilidad de su utilización. El propósito de las observaciones clínicas es único, ya que estas proporcionan información acerca del funcionamiento de sistemas sensoriales específicos que no se pueden evaluar de otra for- ma, principalmente información acerca de las funciones vestibulares, propioceptivas y vestibulares-propioceptivas (Blanche \& Reinoso, 2008). Algunas desventajas en la utilización de las observaciones clínicas, se relacionan con la necesidad de contar con habilidades de razonamiento clínico y por lo tanto su interpretación depende de los conocimientos específicos del terapeuta.

Como la comprensión de la teoría de integración sensorial ha evolucionado, también lo han hecho las observaciones que se utilizan en la práctica clínica. Algunos autores han intentado organizar estas observaciones mientras otros han estudiado el desempeño de los niños típicos y proporcionado normas que se utilizan para su interpretación. Por ejemplo, Fisher \& Smith Roley (1991) y Blanche (2002/2010) organizaron el método de recopilación de información sobre el procesamiento sensorial y signos neurológicos blandos que dependen del razonamiento clínico, así como las normas existentes para interpretarlos. Por otro lado Wilson, Pollok, Kaplan \& (2000) han establecido normas para niños en seis de estas tareas que se pueden utilizar para identificar signos específicos de retraso motor. Otros autores han estudiado observaciones específicas. Por ejemplo, Petri y Anderson (1980), estudiaron los movimientos oculares y cefálicos; Gilliam, Mayberry, Steward, Kenyon y Gaebler (1981) investigaron los movimientos oculares en niños típicos; Harris (1981) estudió la duración y la calidad de la extensión en posición prona; Dunn (1981) examinó el desempeño de un grupo de niños en edad pre-escolar; Fraser (1986) estudio la flexión en contra la gravedad en posición supina; Gregory Flock y Yerxa (1984) estandarizaron la administración de la extensión en contra de la gravedad en posición prona; Fisher (1989) estudió la relación de las reacciones de equilibrio con la integración sensorial; y Magalhaes, Koomar y Cermak, (1989) estudiaron la capacidad de los niños típicos para ejecutar una serie de saltos y su relación con la coordinación motora bilateral.

En el estudio que presentamos a continuación proporcionamos información preliminar sobre el funcionamiento de niños típicos entre 5 a 7.11 años de edad en un grupo de observaciones centradas en aspectos del control postural, planeamiento motor y control motor relacionadas al procesamiento sensorial. De esta manera, el terapeuta puede comparar los resultados de sus usuarios con otros niños de la misma edad.

Proporcionar normas de las observaciones más comúnmente utilizadas es importante para la práctica clínica porque proporcionan una estructura para el proceso de interpretación. 
El objetivo de este estudio fue desarrollar un sistema estandarizado de recoger información en las siguientes pruebas: Equilibrio en un pie, extensión antigravitatoria, flexión antigravitatoria, movimientos secuenciales de los dígitos, diadochokinesis; determinar la confiabilidad entre 4 evaluadores independientes; y finalmente medir la respuesta de niños típicos y atípicos de 5 a 7 años y 11 meses en el grupo de observaciones clínicas seleccionadas para este estudio.

\section{MÉTODO}

El estudio tuvo los siguientes pasos: Desarrollo de la herramienta, estimación de confiabilidad de la mediciones; recolección de datos en niños entre 5 y 7 años y 11 meses; recolección de datos en niños que fueron identificados con disfunciones de integración sensorial; y análisis de datos. En este artículo presentamos los resultados preliminares en 5 pruebas con valor discriminatorio: equilibrio en un pie, extensión en prono, flexión en supino, secuenciación de dedos, y diadochokinesis.

\section{Desarrollo de la Herramienta}

Para estandarizar las observaciones estructuradas en una muestra chilena, se utilizó el protocolo de OBSERVACIONES ESTRUCTURADAS DEL DESEMPEÑO MOTOR EN RELACIÓN CON LOS SISTEMAS SENSORIALES, desarrollado por Imperatore Blanche, Reinoso, Kiefer-Blanche, (versión 3, 2010 ) basado en Ayres (1972, 1984). La validez de contenido se estableció revisando el protocolo original de Ayres (1984) y haciendo una revisión de la literatura de signos neurológicos blandos que podrían estar relacionados a procesamiento vestibular y propioceptivo y dificultades motrices en niños. De esta revisión de desarrolló un sistema específico de recolección de datos que incluye las siguientes pruebas: prueba de Schilder's, equilibrio en un pie, extensión y flexión antigravitatoria, secuenciación de pulgar a dígitos, diadochokinesis, saltos (polichinela), reacción a input vestibular gravitatorio entre otras pruebas. En este artículo se presentan los datos de 5 pruebas: Equilibrio en un pie, extensión antigravitatoria en prono, flexión antigravitatoria en supino, secuenciación de dígitos y diadochokinesis. Una muestra de estas observaciones y la información que proporcionan se detallan en la tabla 1.

Tabla 1

Observaciones clínicas estructuradas seleccionadas en el presente estudio.

\begin{tabular}{|c|c|}
\hline Prueba & Funciones Relacionadas \\
\hline Equilibrio en un pie & $\begin{array}{c}\text { Funciones posturales relacionadas a sistemas vestibulares y propioceptivas se } \\
\text { observan en la mantención de la postura de equilibrio en pie con ojos abiertos } \\
\text { y con ojos cerrados. }\end{array}$ \\
\hline Moviemientos secuenciales de los dígitos & $\begin{array}{c}\text { Funciones propioceptivas y de planeamiento motor se observan en la fluidez } \\
\text { de movimiento, la presión de cada dedo y la presencia de movimientos asocia- } \\
\text { dos, al tocar consecutivamente cada uno de los dedos con el pulgar. }\end{array}$ \\
\hline Extensión en Prono & $\begin{array}{c}\text { Funciones propioceptivas y de planeamiento motor se observan en la ritmici- } \\
\text { dad del movimiento y presencia/ausencia de movimientos asociados, al eje- } \\
\text { cutar movimientos secuenciales de antebrazos. }\end{array}$ \\
\hline Flexión en Supino & $\begin{array}{c}\text { Control postural y extensión antigravitatoria relacionada a funciones vestibula- } \\
\text { res se observan en la capacidad de llevantar simultáneamente el tronco y los } \\
\text { miembros superiores e inferiores de la superficie de apoyo, de la mantención } \\
\text { de esta posición y del esfuerzo requerido }\end{array}$ \\
\hline & $\begin{array}{c}\text { Control postural y flexión antigravitatoria relacionada a procesamiento somato- } \\
\text { sensorial y vestibular que se observa en la elevación simultánea de las extremi- } \\
\text { dades superiores e inferiores, de la mantención de la cabeza en la línea media, } \\
\text { y de la cantidad de tiempo que puede permanecer en esta posición. }\end{array}$ \\
\hline
\end{tabular}




\section{Confiabilidad de las mediciones}

Cuatro evaluadores participaron en un estudio de confiabilidad entre examinadores independientes, para lo cual se observaron videos de la evaluación de 3 niños, a los cuales se les otorgaron puntajes específicos durante el desempeño de las diferentes pruebas. Se presentan las pruebas con valores aceptables de confiabilidad así como también las que presentan mayor validez clínica en la sección de resultados.

\section{Recolección de datos en niños entre 5 y 7 años 11 meses}

Se entrenaron a cuatro examinadores que recolectaron los datos en 90 niños entre 5 y 7 años, 11 meses. Se incluyó en esta evaluación, a un grupo de niños provenientes de varios establecimientos educacionales y de diferentes estratos económicos, incluyendo jardín infantil municipal y particular, colegio particular subvencionado, municipal y particular pagado, ubicados dentro del área metropolitana, en la ciudad de Santiago. La mayoría de los participantes fueron seleccionados por sus profesores, considerando que presentaban rendimiento y conducta acorde a lo esperado para su edad. Fueron excluidos de esta muestra niños que pre- sentaran antecedentes de retraso en el desarrollo o algún problema neurológico. El 90\% de las evaluaciones fueron realizadas en los establecimientos educacionales, a los cuales asistían. Los padres de los niños autorizaron la participación de sus hijos a través de un consentimiento informado. La evaluación se realizó de manera individual, en un tiempo de aproximadamente 60 minutos. La muestra estuvo constituida por un grupo de 44 niños y 46 niñas distribuidos de la siguiente manera: 15 niños y 15 niñas entre 5.0 y 5.11 años; 15 niños y 15 niñas entre 6.0 y 6.11 años; 14 niños y 16 niñas entre 7 y 7.11 años. Los resultados se ingresaron en Qualtrics y se conviertieron en una tabla Excel para su posterior análisis. Los niños fueron divididos por edad y por nivel socio-económico, considerando como bajo nivel socio económico a los que asistian a colegios y jardines particular subvencionado /municipalizados y alto nivel socioeconomico a niños que asistian a colegios particulares pagados.

Se evaluaron además 26 niños identificados con problemas de procesamiento sensorial por profesionales con formación en Integración sensorial con el propósito de estudiar la validez discriminativa de las pruebas estudiadas. En este artículo se presentan las pruebas con mayor validez discriminativa y con mayor confiabilidad.

Tabla 2

Muestra de niños de 5.0 a 7.11 años de edad evaluada en el presente estudio.

\begin{tabular}{|c|c|c|c|c|c|}
\hline Grupo & $5.0-5.11$ & $6.0-6.11$ & $7.0-7.11$ & $\mathrm{n}$ & $\mathrm{M}$ \\
\hline Atípicos & 7 & 10 & 9 & 26 & 6.5 \\
\hline Típicos & 30 & 30 & 30 & 90 & 6.4 \\
\hline Atípicos + Típicos & 37 & 40 & 39 & 116 & 6.4 \\
\hline
\end{tabular}

Nota. $\mathrm{n}=$ Número de niños evaluados por grupo etario; $\mathrm{M}=$ edad promedio. 


\section{Resultados}

Los valores de confiabilidad entre examiandores independientes se ilustran en la Tabla 3. Los valores obtenidos en las cinco pruebas en niños de 5 a 7 años y 11 meses y sus valores de discriminación en niños identificados con y sin problemas. La confiabilidad entre examinadores independientes se estudió con cuatro evaluadores que observaron videos de tres niños desempeñando cada prueba. Siguiendo las recomendaciones de Shrout and Fleiss (1979) para las mediciones de coeficientes entre clases (ICC) y el coeficiente Kappa para la medición de fluidez (dicotómica). Se obtuvieron resultados que fluctuaron entre 0.33 y 0.99 . Para obtener normas, se utilizaron estadísticas descriptivas para obtener el puntaje promedio y la desviación stan- dard de cada medida basado en la edad del niño. La validez discriminatoria se obtuvo usando $T$ test comparando diferentes muestras por su nivel cronológico. Los valores discriminativos resultaron estadísticamente significativos en diferentes grupos $(p=.05 ; p=.001)$ (Blanche, Reinoso, Kiefer-Blanche \& Barros, 2016).

\section{Confiabilidad entre evaluadores independientes}

Las variables fueron analizadas individualmente para determinar su el inter-acuerdo entre los diferentes examinadores independientes. En la tabla 3 se presentan los resultados del estudio de confiabilidad entre evaluadores para cada una de las pruebas administradas.

Tabla 3

Acuerdo entre evaluadores independientes

\begin{tabular}{|c|c|c|c|}
\hline Prueba & Shrout \& Fleiss & Kappa & Poder de acuerdo* \\
\hline Equilibrio en un pie & 0.98 & NA & Casi Perfecto \\
\hline Extension en Prono (tiempo) & 0.33 & NA & Apropriado \\
\hline Flexion en Supino & 0.99 & NA & Casi Perfecto \\
\hline Secuenciacion de dígitos & 0.49 & NA & Moderado \\
\hline Secuenciación de dígitos (Fluidez) & NA & 1.00 & Perfecto \\
\hline Diadochokinesis & 0.76 & NA & Substancial \\
\hline
\end{tabular}

Nota. ${ }^{*}<0.00=$ pobre; $0-0.20=$ escazo; $0.21-0.40=$ apropiado; $0.41-0.60$ = moderado; $0.61-0.80$ substancial; 0.81 -1.00 Casi perfecto, perfecto.

\section{Datos para la interpretación de las observaciones clínicas}

Las siguentes tablas proporcionan los resultados obtenidos en equilibrio en un pie. De este modo, la Tabla
4 reporta los valores obtenidos (promedio y desviación estándar) en la muestra para la prueba equilibrio en un pie en sus diferentes condiciones de valoración. 
Tabla 4

Equilibrio en un pie - mejor puntaje de dos intentos

\begin{tabular}{|c|c|c|c|c|c|c|c|c|}
\hline \multirow[t]{2}{*}{ Edad } & \multicolumn{2}{|c|}{$\begin{array}{l}\text { Pie derecho, } \\
\text { ojos abiertos }\end{array}$} & \multicolumn{2}{|c|}{$\begin{array}{l}\text { Pie derecho, } \\
\text { ojos cerrados }\end{array}$} & \multicolumn{2}{|c|}{$\begin{array}{c}\text { Pie izquierdo, ojos } \\
\text { abiertos }\end{array}$} & \multicolumn{2}{|c|}{$\begin{array}{c}\text { Pie izquierdo, ojos } \\
\text { cerrados }\end{array}$} \\
\hline & $M$ & SD & $M$ & SD & $M$ & SD & $M$ & SD \\
\hline $5.0-5.11$ & 10.0 & 4.8 & 4.5 & 3.5 & 8.5 & 3.9 & 3.4 & 1.9 \\
\hline $6.0-6.11$ & 15.5 & 4.7 & 5.7 & 4.2 & 15.6 & 5.5 & 6.0 & 5.0 \\
\hline $7.0-7.11$ & 16.6 & 5.0 & 8.0 & 5.6 & 16.5 & 4.9 & 7.2 & 5.4 \\
\hline
\end{tabular}

Nota. $\mathrm{M}=$ Promedio. SD = Desviación Estándar. Todos los puntajes reportados en segundos.

La tabla 5 detalla los valores obtenidos para la extensión y flexión en contra de la gravedad. Se reporta el mejor valor obtenido en dos intentos.

Tabla 5

Extensión y flexión en contra la gravedad, mejor tiempo de dos intentos.

\begin{tabular}{|c|c|c|c|c|}
\hline Edad & \multicolumn{2}{|c|}{ Extensión anti gravedad } & \multicolumn{2}{c|}{ Flexión anti gravedad } \\
\hline & M & SD & M & SD \\
\hline $5.0-5.11$ & 16.0 & 10.2 & 24.9 & 19.7 \\
\hline $6.0-6.11$ & 20.2 & 11.5 & 33.2 & 19.9 \\
\hline $7.0-7.11$ & 21.2 & 10.6 & 44.3 & 17.6 \\
\hline
\end{tabular}

Nota. $M$ = Promedio; SD = Desviación Estándar. Todos los puntajes reportados en segundos.

La tabla 6 ilustra los valores obtenidos en las pruebas de secuenciación de dígitos (oposición al pulgar) para la mano derecha e izquierda y diadochokinesis para ambas extremidades superiores (promedio y desviación estándar).

Tabla 6

Secuenciación de dedos y diadochokinesis

\begin{tabular}{|c|c|c|c|c|c|c|c|c|}
\hline \multicolumn{4}{|c|}{ Toque secuencial de los dígitos } & \multicolumn{4}{c|}{ Diadochokinesis } \\
\hline Edad & \multicolumn{2}{|c|}{ Mano D* } & \multicolumn{2}{c|}{ Mano I** } & \multicolumn{2}{c|}{ Mano D } & \multicolumn{2}{c|}{ Mano I } \\
\hline & M & SD & M & SD & M & SD & M & SD \\
\hline $5.0-5.11$ & 4.9 & 2.2 & 4.8 & 2.5 & 4.8 & 1 & 4.6 & 1.2 \\
\hline $6.0-6.11$ & 5.3 & 2.3 & 5.7 & 2.9 & 5.3 & 1.1 & 5 & 1 \\
\hline $7.0-7.11$ & 7.4 & 2.1 & 7.3 & 2.6 & 6.1 & 1.1 & 5.7 & 1.1 \\
\hline
\end{tabular}

Note. $\mathrm{D}=$ Derecha; I = Izquierda; $\mathrm{M}$ = Promedio; SD = Desviación Estándar;

$(*)=$ Mejor puntaje de dos intentos. Todos los puntajes son reportados en segundos. 
Los valores normativos de estas pruebas confirman el desarrollo de niños entre 5 y 7.11 años en las areas de control postural y planeamiento motor. Las cinco pruebas evaluadas muestran un incremento en el funcionamiento sensorio-motor a través del desarrollo. Las habilidades evaluadas en estas pruebas parecen dominarse con el crecimiento y desarrollo del niño ilustrados por la propensidad de los valores a disminuir en su dispersión (desviación estándar).

\section{Valores discriminativos}

Valores discriminativos se midieron en las siguientes pruebas: equilibrio en un pie, extensión antigravitatoria, flexión antigravitatoria, secuenciación de dedos y diadochokinesis. Los resultados preliminares entre niños separados en dos grupos como típicos $(\mathrm{N}=90)$ y niños atípicos $(\mathrm{N}=26)$ referidos a servicios de Terapia Ocupacional (no necesariamente por problemas motores) varían entre $p=.00$ y $p=.43$ lo que sugiere que algunas de estas pruebas tienen mejor validez discriminatoria que otras. Estos y otros resultados se publicarán separadamente (Blanche, Reinoso, Kiefer-Blanche, Barros, en preparacion).

\section{Discusión}

Los resultados preliminares de este estudio sugieren que las cinco pruebas reportadas se pueden utilizar en Chile para identificar niños con dificultades sensoriomotoras. Es más, si estas pruebas se combinan con otras pruebas de procesamiento vestibular y propioceptivo (ejemplo: prueba del Nistagmos Post-rotatorio (Ayres, 1981), y Observaciones Clínicas de Propiocepción (Blanche, Bodison, Chang \& Reinoso, 2012; Blanche, Reinoso, Chang \& Bodison, 2012), este grupo de observaciones pueden esclarecer las áreas de disfunción relacionadas a estos sistemas.

Los resultados preliminares sugieren además que este grupo de cinco observaciones son válidas y confiables. La validez está establecida por numerosas revisiones de la literatura realizada por los autores y reportadas por investigadores independientes en contextos internacionales. De este modo, algunos investigadores han reportados tendencias del desarrollo, valores típicos y diferencias entre grupos etarios pero esa informa- ción es escasa en contextos de habla hispana. La confiabilidad examinada en 4 observadores entrenados e independientes sugieren puntajes de coeficientes entre clases (ICC) entre 0.33 y 0.99 ; valores que oscilan entre "apropiados" y "casi perfectos/perfectos" en su extremos bajos y altos, respectivamente. Un aspecto cualitativo dicotómico fue analizado individualmente (fluidez durante el toque secuencial de los dígitos) utilizando un coeficiente Kappa que indica una fuerza de acuerdo "perfecta" entre los examinadores independientes. Estos valores indican que diferentes examinadores, con entrenamiento apropiado en estas observaciones, pueden reproducir puntajes estables y adecuados para su utilización en contextos de clínica e investigación.

Los resultados también sugieren que las habilidades de control postural y planeamiento motor en niños entre 5 y 7 años 11 meses evolucionan y que el funcionamiento en estas pruebas es diferente en niños con y sin problemas (Blanche, Reinoso, Kiefer-Blanche \& Barros, 2016).

Para su aplicación clínica recomendamos la adherencia, entrenamiento formal y práctica en los procedimientos de administración para garantizar la integridad y confiabilidad de los datos. El proceso de interpretación clínica en estas observaciones es complejo y debe ser guiado por el conocimiento actualizado en la teoría de integración sensorial y otras pruebas administradas. Se presentan tablas con datos individuales con la información de tendencia central y dispersión para su utilización de acuerdo a la fórmula: $Z=X-M / S D$.

\section{CONCLUSIÓN}

La importancia de este estudio se relaciona a tres areas específicas: (1) proprociona información sobre el comportamiento en estas pruebas en niños Chilenos; (2) suministra tendencias relacionadas al funcionamiento de niños entre 5 años y 7 años 11 meses que posibilitan interpretar si el funcionamiento de un niño durante una evaluación está dentro de lo que se espera para esa edad; (3) ofrece información sobre la validez discriminativa de estas pruebas.

Las observaciones clínicas son necesarias en toda evaluación de niños en los cuáles se sospechan alteraciones del desarrollo o disfunción y son centrales a la teoría de integración sensorial, ya que los constructos explorados se relacionan teóricamente a áreas del control postural, planeamiento y control motor. 
Estudios futuros pueden esclarecer la relación entre diferentes pruebas administradas ante la presencia de disfunción o desviación del desarrollo o situación de riesgo o concurrentemente con otras pruebas de medición. La inclusión de niños en otros grupos etarios y sus parámetros podrían determinar tendencias del desarroIlo y dificultad en estas observaciones. La asociación y síntesis de variables también podría explorarse y establecer su relación a la teoría de integración sensorial en el contexto Latinoamericano.

\section{REFERENCIAS BIBLIOGRÁFICAS}

Ayres, A. J. (1984). Personal communication.

Ayres, A. J. (1972). Sensory integration and learning disorders. Western Psychological Services. Los Angeles, CA.

Adams, R.M.; Jenci, J.K.; Estes, R.E. (1974). Soft neurological signs in learning disabled

children and controls. American Journal of Disabled Children, 128, 614618.

Blanche, E. I. (2002/2010). Observations based on sensory integration. (video and book). Pediatric Therapy Network. Torrance, CA.

Blanche, E. I. and Reinoso, G. (2008). The use of clinical observations to evaluate

proprioceptive and vestibular functions. The American Occupational Therapy Association, Continuing Education Article (AOTA, CEU). The American Occupational Therapy Association, OT Practice.

Blanche, E.I., Reinoso, G., Kiefer-Blanche, D., \& Barros, A. (2016). Manuscript in preparation.

Blanche, E.I., Bodison, S., Chang, M.C and Reinoso, G. (2012). Development of the

comprehensive observations of proprioception (COP): Validity, reliability and factor analysis. American Journal of Occupational Therapy, 66(6): 691-698. doi. 10.5014/ajot.2012.003608

Blanche, E.I., Reinoso, G., Chang, M.C and Bodison, S. (2012). Proprioceptive processing difficulties among children with autism spectrum disorders and developmental disabilities. American Journal of Occupational Therapy, 66(5): 621-624. doi 10.5014/ ajot.2012.004234

Dunn, W. (1981). A Guide to Testing Clinical Observations in Kindergarteners: American

Occupational Therapy Association. Rockville, MD.

Fellick, J.M., Thomson, A.P., Sills, J., and Hart, C.A. (2001). Neurological soft signs in

mainstream pupils. Archives of disease in childhood, 85(5): 371-374.

Fisher, A.G. (1989). Objective measurement of the quality of response during two equilibrium tests. Physical and Occupational Therapy in Pediatrics. 9, 57-78.

Fisher, A. and Smith Roley, S. (1991). Clinical observations. Unpublished document. United

States.

Fraser, A. (1986). Standardization of the supine flexion test in children ages 4 through 8. Unpublished master's thesis, University of Southern California, Los Angeles, CA.

Gillberg, C., Kadesjo, B. (2003). Why bother about clumsiness? The implications of having

developmental coordination disorder (DCD). Neural Plasticity 1003; 10: 59-68.

Gilligan, M.B., Mayberry, W., Steward, L., Kenyon, T., \& Gaebler, C. (1981). Measurement of

Ocular Pursuits in Normal Children. American Journal of Occupational Therapy. 35, 249-255.

Gregory-Flock, J.L. \& Yerxa, E.J. (1984). Standardization of the Prone Extension Postural Test

on Children ages 4 through 8. American Journal of Occupational Therapy, 35, 187-194.

Gustafsson, P., Svedin, C.G., Ericsson, I., Karlsson, M.K., Thernlund, G. (2010). Reliability and

validity of the assessment of neurological soft-signs in children with and without attention-deficit hyperactivity disorder. Developmental Medicine and Child Neurology, 52(4): 364-70.

Guftafsson, P., Thernlund, G., Ryding, E., Rosen, I., Cerdblad, M. (2000). Associations between

cerebral blood-flow measured by single photon emission computed tomography (SPECT), electro-encephaloghram (EEG), behavior symptoms, cognition and neurological soft signs in children with attention-deficit hyperactivity disorder (ADHD). Acta Paediatrica, 89(7): 830-835.

Harris, N.P. (1981). Duration and Quality of the Prone Extension Position in four-, six-, and

eight-year-old Normal Children. American Journal of Occupational Therapy, 35: 26-30.

Holden, E.W., Tarnowski, K.J. and Prinz, R.J. (1982). Reliability of neurological soft signs in

children: reevaluation of the PANESS. Journal of Abnormal Child Psychology, 10(2): 163-172.

Jones, V. and Prior, M. (1985). Motor imitation abilities and neurological signs in autistic

children. Journal of Autism and Developmental Disorders, 15(1):37-46.

Magalhaes, L.C., Koomar, J.A., Cermark, S.A. (1989). Bilateral Motor Coordination in 5-to 9-

year-old children: A pilot Study. American Journal of Occupational Therapy, 43, (7), 437-443.

Petri, J. \& Anderson, M. (1980). Eye and Head Movements in ReadingDisabled and Normal 
Children. American Journal of Occupational Therapy, 38 (10), 647-659.

Schaffer, D., O'Connor, P.A., Schafer, S.Q., Prupil, S. (1984). Neurological "soft signs": Their origins and significance for behavior. In Rutter, M. (Ed.), Developmental Neuropsychiatry. London: Churchill Livingstone, 144-163.

Schrout, P.E. and Fleiss, J.L. (1979). Intraclass correlations: Uses in Assessing Rater Reliability. Psychological Bulleting, 86(2): 420-428.

Wilson, B.N., Pollock, N. Kaplan, B.J., Law, M. (2000). Clinical observations of Motor and

Postural Skills. 2nd edition. Framingham, MA. Therapro, Inc. 